\title{
Thermal decoupling of WIMPs: A link between particle physics properties and the small-scale structure of (dark) matter
}

\author{
Torsten BRINGMANN*, II. Institute for Theoretical Physics, University of Hamburg, \\ Luruper Chausse 149, D-22761 Hamburg, Germany \\ E-mail: torsten.bringmann@desy.de
}

\begin{abstract}
The kinetic decoupling of weakly interacting massive particles (WIMPs) in the early universe sets a scale that can directly be translated into a small-scale cutoff in the spectrum of matter density fluctuations. I present a formalism which allows to describe this decoupling process in great detail and to determine the cutoff scale to a high accuracy; with decoupling temperatures of several $\mathrm{MeV}$ to a few $\mathrm{GeV}$, depending on the details of the underlying WIMP microphysics, the smallest protohalos to be formed range between $10^{-11}$ and almost $10^{-3}$ solar masses. Observational consequences and prospects to probe this small-scale cutoff, which would provide a fascinating new window into the particle nature of dark matter, are briefly discussed.
\end{abstract}

Cosmic Radiation Fields: Sources in the early Universe

November 9-12, 2010

Desy, Germany

*Speaker. 


\section{Introduction}

Weakly interacting massive particles (WIMPs) arise in many extensions to the standard model of particle physics (SM) and provide a very well-motivated class of candidates for the so far still elusive nature of the dark matter (DM). The reason for this is the well known fact that WIMPs can be thermally produced in the early universe; the resulting relic density will then generically be of the required order of magnitude if new physics is introduced at the electroweak scale, aiming to solve the shortcomings of the SM from a purely particle physics point of view. Another very appealing consequence of WIMPs is of course that such DM candidates would not only interact gravitationally but could, in principle, also be detected in collider, direct or indirect DM searches.

The basis for the thermal production of DM in the early universe are (co-)annihilations of WIMPs into SM particles and the reverse process of WIMP creation from the annihilation of SM particles. Before chemical freezeout, these processes ensure that the WIMP number density is given by its value in thermal equilibrium, i.e. the Maxwell-Boltzman distribution; once the annihilation rate falls behind the expansion rate of the universe, however, the co-moving WIMP number density stays essentially constant ("freezes out") until today - which makes it straightforward to calculate the resulting DM relic density.

A less often appreciated fact is that WIMPs actually stay in thermal contact with the heat bath of SM particles quite long after these number density changing processes have ceased $[1,2]:$ scattering events between WIMPs and SM particles are much more frequent than WIMP annihilations simply because SM particles are much more abundant (SM particle annihilations to WIMPs, on the other hand, are kinematically possible only in the heavily suppressed tail of the energy distribution). Only when even these processes stop to be efficient after kinetic decoupling, there are no longer any interactions between WIMPs and SM particles and the former have completely decoupled from the thermal bath.

Before kinetic decoupling, any density perturbation in the DM component is tightly bound to density perturbations in the radiation component; only afterwards can DM perturbations freely develop, with free streaming effects setting a lower bound to the size of over-dense regions that will eventually collapse under the influence of gravitation [3, 4, 5]. Since the time of kinetic decoupling critically depends on the detailed DM particle physics properties, the low-mass cutoff in the power spectrum of matter density perturbations provides - at least in principle - a very interesting window into the nature of DM which is quite complementary to other means of DM detection.

\section{Kinetic freeze-out in detail}

The evolution of the WIMP phase-space density $f$ in the early universe is governed by the Boltzmann equation which in a flat Friedmann-Robertson-Walker spacetime reads

$$
E\left(\partial_{t}-H \mathbf{p} \cdot \nabla_{\mathbf{p}}\right) f=C[f],
$$

where $H=\dot{a} / a$ is the Hubble parameter, $a$ the scale factor and $C[f]$ the source term (which describes any changes in $f$ that is not only due to the expansion of the universe). Integration over the WIMP momenta $\mathbf{p}$ brings it into the familiar form

$$
\partial_{t} n_{\chi}+3 H n_{\chi}=a^{-3} \partial_{t}\left(a^{3} n_{\chi}\right)=-\langle\sigma v\rangle\left(n_{\chi}^{2}-n_{\mathrm{eq}}^{2}\right)
$$


where $n_{\chi}$ denotes the WIMP number density, $n_{\mathrm{eq}}$ its value in chemical equilibrium and $\langle\sigma v\rangle$ the thermally averaged annihilation rate. This equation describes the chemical freeze-out process; the resulting asymptotic value of the co-moving number number density $a^{3} n_{\chi}$ is therefore directly related to the DM relic density.

As was found in [6], the process of kinetic decoupling, on the other hand, can be described to a high accuracy by defining a WIMP "temperature"

$$
T_{\chi} \equiv \frac{1}{3 m_{\chi} n_{\chi}} \int \frac{d^{3} p}{(2 \pi)^{3}} \mathbf{p}^{2} f(\mathbf{p})
$$

and considering the second moment of the Boltzmann equation (2.1) - instead of the first moment as for the derivation of Eq. (2.2). Note that this definition does not make any assumptions about the WIMP distribution $f$; for the case of a thermal distribution, however, of course it agrees with the temperature of the system. The difference between $T_{\chi}$ and the temperature $T$ thus gives an indication of how well the WIMPs are kept in thermal equilibrium.

Multiplying Eq. (2.1) by $\mathbf{p}^{2} / E$, integrating it over $\mathbf{p}$ and keeping only the leading order terms in $\mathbf{p}^{2} / m_{\chi}^{2}$ leads, after a lengthy calculation [7], to

$$
\frac{d y}{d x}=2 \frac{m_{\chi} c(T)}{H \tilde{g}^{1 / 2}}\left(1-\frac{T_{\chi}}{T}\right)
$$

where

$$
\begin{aligned}
x & \equiv m_{\chi} / T \\
y & \equiv m_{\chi} g_{\text {eff }}^{-1 / 2} T_{\chi} / T^{2}, \\
\tilde{g}^{1 / 2} & \equiv g_{\text {eff }}^{1 / 2} /\left(1+\frac{1}{4} \frac{g_{\text {eff }}^{\prime}}{g_{\text {eff }}} T\right),
\end{aligned}
$$

and

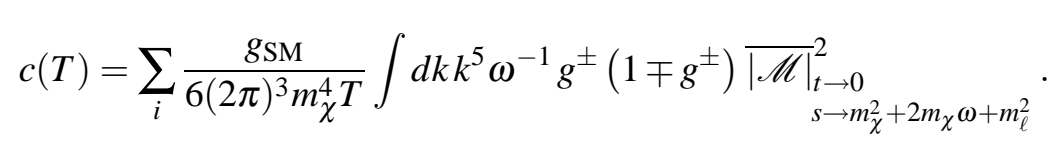

is obtained by summing over all SM scattering partners $i$.

Inspection of Eq. (2.4) shows the expected asymptotic behaviour for the WIMP temperature: at early times, or large $T$, the term in front of the right-hand side is much larger than unity and the solution $T_{\chi}=T$ provides a strong attractor of the differential equation, i.e. the system is very efficiently kept in thermal equilibrium. When $T$ becomes small, the WIMPs finally decouple completely from the thermal bath: the pre-factor becomes negligibly small and $y$ stays constant, i.e. $T_{\chi} \propto T^{2} g_{\text {eff }}^{1 / 2} \propto a^{-2}$. Since the transition between the two regimes happens on a rather short timescale, the kinetic decoupling temperature is naturally defined by equating these two asymptotic behaviours, as if the decoupling process were indeed to occur instantaneously:

$$
x_{\mathrm{kd}}=\left.\frac{m_{\chi}}{T_{\mathrm{kd}}} \equiv g_{\mathrm{eff}}^{1 / 2}\left(T_{\mathrm{kd}}\right) y\right|_{x \rightarrow \infty} .
$$

The above presented formalism allows to calculate the kinetic decoupling temperature to an accuracy of $\mathscr{O}\left(x_{\mathrm{kd}}^{-1}\right)$ and is applicable to any WIMP candidate that was non-relativistic at chemical freeze-out and for which we have $x_{\mathrm{cd}} \ll x_{\mathrm{kd}}$ (while this is typically satisfied, the two freeze-out 
processes may not be so easily distinguishable if the annihilation rate is strongly velocity dependent [8]). As revealed by a large scan over the supersymmetric parameter space, the kinetic decoupling temperature for neutralino DM lies between a few $\mathrm{MeV}$ and a few $\mathrm{GeV}$, with $x_{\mathrm{kd}}$ between 200 and almost $10^{5}$ - a much larger range than for $x_{\text {cd }}$ which falls into the range $20 \lesssim x_{\text {cd }} \lesssim 28$ for neutralinos with the correct relic density [7]. This is a similar range to what was found earlier by using an order-of-magnitude estimate for the kinetic decoupling temperature [9] and indicative of the range of decoupling temperatures generally to be expected for WIMP DM.

\section{The smallest protohalos}

Before kinetic decoupling, WIMPs are tightly coupled to the heat bath, so any density perturbation in the DM component would decay rapidly. Afterwards, DM density perturbations can freely grow under the influence of gravitation; close to kinetic decoupling, however, first a remaining viscous coupling between the DM and radiation fluids and then the free-streaming of the WIMPs generate an exponential cutoff in the power spectrum $[3,10]$. The mass contained within a region the size of the corresponding cutoff-scale is given by

$$
M_{\mathrm{fs}} \approx 2.9 \times 10^{-6}\left(\frac{1+\ln \left(g_{\mathrm{eff}}^{1 / 4} T_{\mathrm{kd}} / 50 \mathrm{MeV}\right) / 19.1}{\left(m_{\chi} / 100 \mathrm{GeV}\right)^{1 / 2} g_{\text {eff }}^{1 / 4}\left(T_{\mathrm{kd}} / 50 \mathrm{MeV}\right)^{1 / 2}}\right)^{3} M_{\odot} .
$$

Acoustic oscillations lead to a similar exponential cutoff in the power spectrum [11, 12] and the characteristic damping mass in this case is

$$
M_{\mathrm{ao}} \approx 3.4 \times 10^{-6}\left(\frac{T_{\mathrm{kd}} g_{\mathrm{eff}}^{1 / 4}}{50 \mathrm{MeV}}\right)^{-3} M_{\odot} .
$$

Which of these two damping mechanisms is more efficient depends on the specific combination of $m_{\chi}$ and $T_{\mathrm{kd}}$. The actual cutoff in the power spectrum is thus given by

$$
M_{\mathrm{cut}}=\max \left[M_{\mathrm{fs}}, M_{\mathrm{ao}}\right],
$$

corresponding to the mass of the smallest gravitationally bound objects that will form much later, during the matter-dominated era, when the primordial density fluctuations enter the non-linear regime. For the neutralino, the mass of these smallest protohalos can be anything between $10^{-11}$ and almost $10^{-3}$ solar masses [7], depending on the supersymmetric model parameters. Another interesting DM candidate is the lightest Kaluza-Klein particle (LKP) in theories with universal extra dimensions, where most recent calculations indicate that the LKP mass has to be $\sim 1.4 \mathrm{TeV}$ in order to satisfy the relic density constraint [13]. In this case, the range of the smallest protohalo masses would be rather small, with $M_{\text {cut }} \sim 10^{-6} M_{\odot}$.

\section{Observational prospects}

An important implication of a clumpy halo for indirect DM searches is that the annihilation signal will be enhanced with respect to a smooth halo since $\left\langle\rho_{\chi}^{2}\right\rangle>\left\langle\rho_{\chi}\right\rangle^{2}$. This enhancement, 
or "boost factor", receives roughly the same contribution from each decade in the distribution of subhalo masses. Even though a reliable calculation of the boost factor goes beyond our current understanding of structure formation, one will eventually need a good estimate for the subhalo cutoff mass in order to perform this task.

Even more interesting is of course the question whether there are ways to directly probe the cutoff. Even though the smallest clumps should in principle appear as gamma-ray point sources, however, these are unlikely to be resolved [14] - even when looking for signatures connected to the proper motion of these objects $[15,16]$. Gravitational lensing seems to be extremely challenging due to the small size of these objects and the fact that their virial radius is much larger than the Einstein radius. However, it has been proposed that multiple images of time-varying sources in strong lensing systems may probe also very small objects [17]. In fact, even sub-solar objects could create observable strong gravitational lensing events and be used to detect small subhalos close to the cutoff mass[18]. Another promising idea could be to focus on the one-point probability function of the diffuse gamma-ray flux [19]; anisotropy probes like angular correlations in the extra-galactic gamma-ray background, on the other hand, are only sensitive to masses considerably larger than what is expected for $M_{\text {cut }}[20,21,22]$.

For all these considerations, one should keep in mind that numerical $N$-body simulations presently cannot resolve the small scales we are interested in here, so relatively little is known about what happens to the smallest protohalos during structure formation and until today. The most likely scenario, however, seems to be that even though they lose some of their material due to tidal interactions in merger processes or encounters with stars, most of the mass resides in a dense and compact central core that remains intact [23].

\section{Conclusions}

The kinetic decoupling of WIMPs leaves an imprint on the spectrum of matter density fluctuations in the form of a small-scale cutoff that strongly depends on the DM particle properties and falls into the range between $10^{-11}$ and almost $10^{-3}$ solar masses. This cutoff scale can be determined to a high accuracy and provides a fascinating link between structures of cosmic size and the specific characteristics of the DM particle. While there are currently still many unknowns, such as the late-time evolution and survival probabilities of the smallest protohalos, there are interesting proposals how one could probe this cutoff - which would, indeed, constitute a new window into the nature of DM.

\section{References}

[1] C. Schmid, D. J. Schwarz and P. Widerin, Amplification of cosmological inhomogeneities from the QCD transition, Phys. Rev. D 59 (1999) 043517 [arXiv:astro-ph/9807257].

[2] X. 1. Chen, M. Kamionkowski and X. m. Zhang, Kinetic decoupling of neutralino dark matter, Phys. Rev. D 64 (2001) 021302 [arXiv:astro-ph/0103452].

[3] S. Hofmann, D. J. Schwarz and H. Stoecker, Damping scales of neutralino cold dark matter, Phys. Rev. D 64 (2001) 083507 [arXiv:astro-ph/0104173]. 
[4] V. Berezinsky, V. Dokuchaev and Y. Eroshenko, Small-scale clumps in the galactic halo and dark matter annihilation, Phys. Rev. D 68 (2003) 103003 [arXiv:astro-ph/0301551].

[5] A. M. Green, S. Hofmann and D. J. Schwarz, The power spectrum of SUSY-CDM on sub-galactic scales, Mon. Not. Roy. Astron. Soc. 353 (2004) L23 [arXiv:astro-ph/0309621].

[6] T. Bringmann and S. Hofmann, Thermal decoupling of WIMPs from first principles, JCAP 0407 (2007) 016 [arXiv:hep-ph/0612238].

[7] T. Bringmann, Particle Models and the Small-Scale Structure of Dark Matter, New J. Phys. 11 (2009) 105027 [arXiv:0903.0189 [astro-ph.CO]].

[8] J. B. Dent, S. Dutta and R. J. Scherrer, Thermal Relic Abundances of Particles with Velocity-Dependent Interactions, Phys. Lett. B 687 (2010) 275 [arXiv:0909.4128 [astro-ph.CO]].

[9] S. Profumo, K. Sigurdson and M. Kamionkowski, What mass are the smallest protohalos?, Phys. Rev. Lett. 97 (2006) 031301 [arXiv:astro-ph/0603373].

[10] A. M. Green, S. Hofmann and D. J. Schwarz, The first WIMPy halos, JCAP 0508 (2005) 003 [arXiv:astro-ph/0503387].

[11] A. Loeb and M. Zaldarriaga, The small-scale power spectrum of cold dark matter, Phys. Rev. D 71 (2005) 103520 [arXiv:astro-ph/0504112].

[12] E. Bertschinger, The effects of cold dark matter decoupling and pair annihilation on cosmological perturbations, Phys. Rev. D 74 (2006) 063509 [arXiv:astro-ph/0607319].

[13] G. Belanger, M. Kakizaki and A. Pukhov, Dark matter in UED : the role of the second KK level, arXiv:1012.2577 [hep-ph].

[14] L. Pieri, E. Branchini and S. Hofmann, Difficulty of detecting minihalos via gamm rays from dark matter annihilation, Phys. Rev. Lett. 95 (2005) 211301 [arXiv:astro-ph/0505356].

[15] S. M. Koushiappas, Proper motion of gamma-rays from microhalo sources, Phys. Rev. Lett. 97 (2006) 191301 [arXiv:astro-ph/0606208].

[16] S. Ando, M. Kamionkowski, S. K. Lee and S. M. Koushiappas, Can proper motions of dark-matter subhalos be detected?, Phys. Rev. D 78 (2008) 101301 [arXiv:0809.0886 [astro-ph]].

[17] L. A. Moustakas et al., Strong gravitational lensing probes of the particle nature of dark matter, arXiv:0902.3219 [astro-ph.CO].

[18] J. Chen and S. M. Koushiappas, Gravitational Nanolensing from Subsolar Mass Dark Matter Halos, Astrophys. J. 724 (2010) 400 [arXiv:1008.2385 [astro-ph.CO]].

[19] S. K. Lee, S. Ando and M. Kamionkowski, The Gamma-Ray-Flux Probability Distribution Function from Galactic Halo Substructure, JCAP 0907 (2009) 007 [arXiv:0810.1284 [astro-ph]].

[20] S. Ando and E. Komatsu, Anisotropy of the cosmic gamma-ray background from dark matter annihilation, Phys. Rev. D 73 (2006) 023521 [arXiv:astro-ph/0512217].

[21] S. Ando, E. Komatsu, T. Narumoto and T. Totani, Dark matter annihilation or unresolved astrophysical sources? Anisotropy probe of the origin of cosmic gamma-ray background, Phys. Rev. D 75 (2007) 063519 [arXiv:astro-ph/0612467].

[22] M. Fornasa, L. Pieri, G. Bertone and E. Branchini, Anisotropy probe of galactic and extra-galactic Dark Matter annihilations, Phys. Rev. D 80 (2009) 023518 [arXiv:0901.2921 [astro-ph]].

[23] A. Schneider, L. Krauss and B. Moore, Impact of Dark Matter Microhalos on Signatures for Direct and Indirect Detection, Phys. Rev. D 82 (2010) 063525 [arXiv:1004.5432 [astro-ph.GA]]. 